\title{
O uso do método PROMETHEE para seleção de candidatos à bolsa-formação do Pronatec
}

\author{
Vanessa Batista de Sousa Silva ${ }^{a}$, Fernando Schramm ${ }^{\text {b* }}$, Hugo Riccely Cunha de Carvalho \\ avbschramm@gmail.com, UATEC, Brasil \\ b*fernandoschramm@globo.com, UAEP, Brasil \\ chugoriccely@hotmail.com, CSTT, Brasil
}

\begin{abstract}
Resumo
O objetivo deste estudo é a construção de um procedimento para apoiar as unidades do Senai em Campina Grande$\mathrm{PB}$, na seleção de alunos do ensino médio para cursos de educação profissional e tecnológica disponibilizados pelo governo federal através do Pronatec. 0 procedimento inclui o estabelecimento dos critérios de exclusão e seleção dos candidatos, conforme as diretrizes do Pronatec e regras estabelecidas pela Secretaria de Educação do Estado; a avaliação estruturada dos candidatos, onde é utilizado o método multicritério PROMETHEE 11; e a estruturação do processo de divulgação do resultado da seleção. Com critérios bem definidos e com a garantia da consideração de todos eles na avaliação dos candidatos foi possivel, através do procedimento, realizar uma seleção melhor dos candidatos que atendesse tanto aos critérios sociais estabelecidos pelo programa como também ao perfil demandado por esse tipo de formação e pelo setor produtivo.
\end{abstract}

Palavras-chave

Análise multicritério. PROMETHEE. Seleção de candidatos. Pronatec.

\section{Introdução}

Em 2011, o governo brasileiro criou o Programa Nacional de Acesso ao Ensino Técnico e Emprego (Pronatec), que corresponde a um conjunto de ações para expansão e democratização da oferta de cursos de educação profissional e tecnológica, visando suprir o mercado nacional com mão de obra qualificada, essencial para o desenvolvimento econômico do país. 0 programa contempla uma série de subprogramas, projetos e ações de assistência técnica e financeira que juntos oferecerão oito milhões de vagas a brasileiros de diferentes perfis para os próximos quatro anos. 0 programa é realizado em parceria com os estados, Distrito Federal e com os serviços nacionais de aprendizagem, que através de suas organizações e instituições oferece uma série de cursos destinados à formação e qualificação de profissionais demandados pelo setor produtivo, que financia o sistema através de contribuições dos trabalhadores.

Na Paraíba, o Pronatec, por meio da Secretaria de Educação do Estado, disponibilizou, apenas para 2012, mais de seis mil vagas para cursos profissionalizantes em todo o estado, destinadas a estudantes do ensino médio, incluindo alunos do programa de Educação de Jovens e Adultos (EJA), trabalhadores e beneficiários dos programas federais de transferência de renda. Os cursos são oferecidos pelas unidades do Serviço Nacional de Aprendizagem Industrial (Senai) do estado, que também são responsáveis pela seleção dos candidatos. Para selecionar os candidatos, o Senai toma como referências as diretrizes nacionais do Pronatec e critérios específicos estabelecidos pela Secretaria de Educação da Paraíba. No entanto, a seleção é realizada de forma desestruturada através da análise da documentação dos alunos, o que não garante que aspectos específicos do Pronatec sejam levados em consideração em todos os processos seletivos, podendo implicar em uma seleção ruim, que, consequentemente, poderá comprometer as metas do programa.

A realização de um processo seletivo bem estruturado, com critérios bem definidos e a garantia da consideração de todos eles na avaliação dos 
candidatos, garante uma seleção melhor, que, neste caso, corresponde a candidatos que, além de atender aos critérios sociais estabelecidos pelo programa, atende também ao perfil demandado por esse tipo de formação e pelo setor produtivo no qual espera-se o ingresso desses profissionais. Nesse sentido, a análise multicritério de apoio à decisão (MCDA - Multi-Criteria Decision Analysis) se apresenta como uma ferramenta poderosa para auxiliar a seleção de candidatos aos cursos do Senai, particularmente aqueles que ingressam por meio do Pronatec, pois auxilia na estruturação do problema, na avaliação dos candidatos e na seleção propriamente dita, a qual garante a consideração de todos os critérios estabelecidos pelos envolvidos na decisão. Além disso, a técnica ajuda a promover a imparcialidade da decisão pública, a qual é prevista pela Constituição Federal do Brasil, em seu artigo 37.

A MCDA é uma técnica para estruturação e análise de problemas complexos, caracterizados pela existência de múltiplos critérios de decisão, sendo alguns deles conflitantes entre si, e pela produção de ações, cujas consequências têm impacto econômico, social e/ou ambiental. De acordo com Belton e Stewart (2002), MCDA provê a organização e sintetização das informações em um processo decisório, fazendo com que todos aspectos envolvidos na decisão sejam efetivamente levados em consideração. Com isso, é possível garantir mais transparência no processo decisório, que é um aspecto fundamental em decisões com consequências diretas sobre a sociedade (MUNDA, 2008). Viana e Alencar (2012) observaram o aumento do uso dessa técnica em problemas de seleção de fornecedores, a exemplo do trabalho de Schramm e Morais (2012), no qual o método multicritério SMART foi usado para selecionar fornecedores para uma empresa de construção civil. Verifica-se também o uso da técnica para resolver problemas de seleção escolar: com o objetivo de avaliar a qualidade do processo de seleção de uma importante universidade pública do Brasil, Rangel e Gomes (2010) ordenaram os candidatos selecionados com o uso dos métodos multicritérios UTA e UTA-CR, os quais são baseados na teoria da utilidade multiatributo (MAUT - MultiAttribute Utility Theory); Leyva-Lopez (2005) propôs um procedimento para seleção de estudantes que combina o método ELECTRE 111 com algoritmos genéticos.

Neste estudo, MCDA é utilizada para construir um procedimento para apoiar as unidades do Senai em Campina Grande-PB, na seleção de alunos do ensino médio para participarem dos cursos de educação profissional e tecnológica disponibilizados pelo governo federal através do Pronatec, com duração mínima de um ano e com o benefício da bolsa-formação do Pronatec. 0 procedimento inclui o estabelecimento dos critérios de exclusão e seleção dos candidatos, conforme as diretrizes do programa e algumas regras estabelecidas pela Secretaria de Educação do Estado da Paraíba específicas para o Pronatec; a avaliação estruturada dos candidatos, onde é utilizado o método multicritério PROMETHEE 11 (BRANS; VINCKE, 1985) para construção de um ranking de candidatos baseado em seus respectivos desempenhos dentro de cada um dos critérios considerados; e a estruturação do processo de divulgação do resultado da seleção.

0 artigo está dividido em seis seções: a seção 2 apresenta uma visão geral sobre MCDA, enfatizando o estudo da família de métodos multicritérios PROMETHEE; a seção 3 traz o contexto do processo decisório, onde são apresentados os principais fundamentos do Pronatec; a seção 4 mostra o procedimento proposto; a seção 5 apresenta uma aplicação do procedimento e uma análise comparativa entre o processo de seleção convencionalmente realizado pelas unidades do Senai de Campina Grande-PB e o processo de seleção apoiado pelo procedimento proposto; por fim; algumas conclusões são mostradas na seção 6 .

\section{Análise multicritério de apoio à decisão}

Vincke (1992) define um problema multicritério como sendo a situação na qual, existindo um conjunto de ações $A$ e uma família de critérios $F$, o decisor deseja: determinar um subconjunto de ações, consideradas, por ele, como sendo as melhores de $A$ (problemática de escolha); alocar as ações a diferentes categorias definidas a priori a partir de um conjunto de regras aplicáveis ao conjunto A (problemática de classificação); ordenar as alternativas de $A$ da melhor para a pior (problemática de ordenação). Roy (1996) apud Almeida (2013) acrescenta a problemática de descrição, cujo objetivo é apoiar a decisão por meio da descrição das ações e de suas consequências. Além dessas problemáticas, existe o problema de seleção de portfólio, que incorpora restrições ao problema de decisão multicritério (VETSCHERA; ALMEIDA, 2012).

Muitos métodos foram desenvolvidos para apoiar problemas de decisão multicritério. Roy (1985) classifica esses métodos da seguinte forma: (i) métodos de critério único de síntese; (ii) métodos de sobreclassificação; e (iii) métodos interativos, que contemplam etapas de cálculos, fornecendo sucessivas soluções de compromisso, com diálogo com os decisores, que representa uma fonte extra de informação sobre as preferências dos mesmos.

A abordagem critério único de síntese consiste em agregar diferentes pontos de vista em uma única função que, em seguida, é otimizada. Nessa 
abordagem, destaca-se a teoria da utilidade multiatributo, conhecida como MAUT (KEENEY; RAIFFA, 1976), do termo em inglês Multi-Attribute Utility Theory; segundo Almeida (2013), os métodos SMART (EDWARD; BARRON, 1994), AHP (SAATY, 1980) e MACBETH (BANA E COSTA; CORTE; VANSNICK, 2005) também fazem agregação através de um critério único de síntese. A abordagem de sobreclassificação consiste em construir e explorar uma relação, denominada relação de sobreclassificação, que representa as preferências dos decisores; as principais vertentes dessa abordagem são as famílias de métodos PROMETHEE (BRANS; VINCKE, 1985) e ELECTRE (VINCKE, 1992).

Os métodos também podem ser classificados de acordo com o significado das constantes nas funções de agregação. Quando as constantes conduzem a trade-offs entre os critérios, os métodos são compensatórios, permitindo que a desvantagem em alguns critérios seja compensada pela vantagem em outros. Nesses casos, essas constantes recebem a denominação de constantes de escala.

Quando as constantes representam apenas medidas de importâncias relativas dos critérios, os métodos são denominados não compensatórios e a denominação utilizada para elas é peso. Nos métodos não compensatórios não há trade-offs entre os critérios; isto significa que um mau desempenho em um dos critérios não pode ser compensado por um bom desempenho em outro. Os métodos baseados em relações de sobreclassificação são classificados como métodos não compensatórios.

No que concerne aos métodos PROMETHEE, um aspecto importante está relacionado à facilidade dos decisores entenderem os conceitos e parâmetros inerentes aos métodos, o que simplifica o processo de modelagem de preferências e, consequentemente, aumenta a efetividade da aplicação do método multicritério. Esse aspecto representa uma grande vantagem dos métodos PROMETHEE sobre outros métodos de sobreclassificação, tais como os métodos ELECTRE. A família ELECTRE usa os conceitos de concordância e discordância para medir as vantagens e desvantagens entre pares de alternativas; muitas vezes, esses conceitos não são devidamente compreendidos pelos decisores, dificultando a implementação do método (SILVA; MORAIS; ALMEIDA, 2010).

Além disso, os métodos PROMETHEE podem ser facilmente implementados em uma linguagem de computador. Talvez isso justifique o crescente número de aplicações com PROMETHEE. Behzadian et al. (2010) criaram um banco de referência que inclui 217 artigos publicados nos principais periódicos internacionais especializados. Todos os artigos descrevem metodologias e aplicações com
PROMETHEE, as quais foram categorizadas pelo autor em nove áreas diferentes: meio ambiente; hidrologia e recursos hídricos; negócios e finanças; logística e transporte; fabricação e montagem; energia; social; e outros tópicos, que incluem aplicações em medicina, agricultura, educação, design, governo e esportes. Em uma pesquisa sobre o uso de MCDA em problemas de seleção de portfólios, na qual foram enfatizados os métodos de sobreclassificação, Vetschera e Almeida (2012) identificaram que o PROMETHEE é o método mais aplicado a esse tipo de problemática.

0 problema de seleção de candidatos para o Pronatec apresenta uma característica multicritério; além disso, o programa visa priorizar alunos que apresentem melhor desempenho médio, considerando todos os aspectos tratados em suas diretrizes. Portanto, trata-se de um problema de decisão multicritério não compensatório, com problemática de ordenação. Esse problema pode ser tratado com um dos métodos da família PROMETHEE, cujas características citadas acima endossam sua adequação à aplicação proposta. A seção seguinte apresenta a família PROMETHEE.

\subsection{PROMETHEE}

0 ponto de partida dos métodos PROMETHEE é uma matriz de avaliação das alternativas com relação a um conjunto de critérios. Em seguida, uma função de preferência é atribuída a cada um dos critérios. A função de preferência de um critério descreve a forma como a preferência do decisor muda com a diferença entre os níveis de desempenho de duas alternativas nesse critério, $g_{j}(a)-g_{j}(b)$, onde $g_{j}(a)$ representa o desempenho da alternativa a no critério $j$ (BRANS; VINCKE, 1985). 0 Quadro 1 apresenta as funções sugeridas pelos métodos PROMETHEE.

A função de preferência fornece a intensidade de preferência de uma alternativa a sobre outra $b$, com

Quadro 1. Funções de preferência.

\begin{tabular}{|c|c|c|}
\hline $\begin{array}{l}\text { Tipo 1: } \\
\text { Usual }\end{array}$ & $\begin{array}{l}g_{j}(a)-g_{j}(b)>0 \\
g_{i}(a)-g_{i}(b) \leq 0\end{array}$ & $\begin{array}{l}P(a, b)=1 \\
P(a, b)=0\end{array}$ \\
\hline $\begin{array}{l}\text { Tipo 11: } \\
\text { Formato U }\end{array}$ & $\begin{array}{l}g_{j}(a)-g_{j}(b)>q \\
g_{i}(a)-g_{i}(b) \leq q\end{array}$ & $\begin{array}{l}P(a, b)=1 \\
P(a, b)=0\end{array}$ \\
\hline $\begin{array}{l}\text { Tipo 111: } \\
\text { Formato V }\end{array}$ & $\begin{array}{l}g_{j}(a)-g_{j}(b)>p \\
g_{j}(a)-g_{j}(b) \leq p \\
g_{j}(a)-g_{j}(b) \leq 0\end{array}$ & $\begin{aligned} P_{j}(a, b) & =1 \\
P_{j}(a, b) & =\left[g_{j}(a)-g_{j}(b)\right] / p \\
P_{j}(a, b) & =0\end{aligned}$ \\
\hline $\begin{array}{l}\text { Tipo IV: } \\
\text { Níveis }\end{array}$ & $\begin{array}{l}\left|g_{j}(a)-g_{j}(b)\right|>p \\
q<\left|g_{j}(a)-g_{j}(b)\right| \leq p \\
\left|g_{j}(a)-g_{j}(b)\right| \leq q\end{array}$ & $\begin{array}{l}P_{J}(a, b)=1 \\
P_{J}(a, b)=1 / 2 \\
P_{J}(a, b)=0\end{array}$ \\
\hline $\begin{array}{l}\text { Tipo V: } \\
\text { Linear }\end{array}$ & $\begin{array}{l}\left|g_{j}(a)-g_{j}(b)\right|>p \\
q<\left|g_{j}(a)-g_{j}(b)\right| \leq p \\
\left|g_{j}(a)-g_{j}(b)\right| \leq q\end{array}$ & $\begin{array}{l}P_{J}(a, b)=1 \\
P_{J}(a, b)=\left[\left|g_{j}(a)-g_{j}(b)\right|-q\right] /(p-q) \\
P_{J}(a, b)=0\end{array}$ \\
\hline $\begin{array}{l}\text { Tipo Vl: } \\
\text { Gaussiana }\end{array}$ & $\begin{array}{l}g_{j}(a)-g_{j}(b)>0 \\
g_{j}(a)-g_{j}(b) \leq 0\end{array}$ & $\begin{array}{l}\text { A preferência aumenta de } \\
\text { acordo com uma distribuição } \\
\text { normal }\end{array}$ \\
\hline
\end{tabular}

Fonte: Almeida e Costa (2002) 
relação a um dado critério $j$, que é representada por $P(a, b)$. A intensidade de preferência deve ser calculada para cada par de alternativas, considerando todos os critérios. Para a função usual (tipo 1), $P_{j}(a, b)$ assume os valores 0 ou 1. Para as demais funções $P(a, b)$ pode assumir qualquer valor no intervalo $[0,1]$, implicando em uma relação de preferência fuzzy, que permite incorporar aspectos de incerteza no julgamento dos decisores.

0 próximo passo é determinar o índice de preferência para cada par de alternativas, que é dado pela agregação das intensidades de preferências determinadas para todos os critérios, referente ao respectivo par de alternativas. A agregação é feita por uma soma, ponderada pelos pesos atribuídos aos critérios (BRANS; VINCKE, 1985):

$$
\begin{aligned}
& P(a, b)=\frac{1}{W} \sum_{j=1}^{n} w_{j} P_{j}(a, b) \\
& W=\sum_{j=1}^{n} w_{j}
\end{aligned}
$$

onde $w_{j}$ é o peso do critério $j$ e $n$ é o número de critérios.

Finalmente, para cada alternativa, dois índices são calculados a partir dos índices de preferências: o fluxo positivo, $Q^{+}\left(\right.$.), e o fluxo negativo, $Q^{-}$(.). A grandeza $Q^{+}(a)$ expressa o quanto uma alternativa a sobreclassifica todas as outras; quanto maior o fluxo positivo de uma alternativa, melhor ela é. A grandeza $Q(a)$ expressa o quanto uma alternativa $a$ é sobreclassificada pelas demais; quanto menor o fluxo negativo de uma alternativa, melhor ela é. Essas grandezas são usadas para explorar as relações entre as alternativas. Elas são determinadas pelas seguintes expressões, respectivamente:

$$
\begin{aligned}
& Q^{+}(a)=\sum_{a \neq b} \frac{P(a, b)}{m-1} \\
& Q^{-}(a)=\sum_{a \neq b} \frac{P(b, a)}{m-1}
\end{aligned}
$$

onde $m$ é o número de alternativas.

Os seguintes métodos da família PROMETHEE são descritos na literatura (BRANS; VINCKE, 1985; BRANS; VINCKE; MARESCHAL, 1986; ALMEIDA; COSTA, 2002):

- PROMETHEE 1 - Pré-ordem parcial das alternativas, destinada à problemática de ordenação;

- PROMETHEE 11 - Estabelece uma pré-ordem completa entre as alternativas, destinada à problemática de ordenação;
- PROMETHEE 111 - Ampliação da noção de indiferença, tratamento probabilístico dos fluxos (preferência intervalar);

- PROMETHEE IV - Pré-ordem completa ou parcial, destinada à problemática de escolha e ordenação em situações onde o conjunto de soluções viáveis é contínuo;

- PROMETHEE V - Nessa implementação, após estabelecer uma ordem completa entre as alternativas (PROMETHEE 11), são introduzidas restrições, identificadas no problema, para as alternativas selecionadas; incorpora-se uma filosofia de otimização inteira;

- PROMETHEE VI - Pré-ordem completa ou parcial. Problemática de escolha e ordenamento. Destinado às situações em que o decisor não consegue estabelecer um valor fixo de peso para cada critério; e

- PROMETHEE GAIA - Extensão dos resultados do PROMETHEE, através de um procedimento visual e interativo.

Além dos métodos acima, um novo método baseado no PROMETHEE foi proposto para apoiar problemas de seleção de portfólio (ALMEIDA; VETSCHERA, 2012; VETSCHERA; ALMEIDA, 2012). A nova proposta soluciona alguns problemas do PROMETHEE $V$, que é a versão da família destinada especificamente a tratar de problemas de seleção de portfólio.

No PROMETHEE 11 uma pré-ordem completa das alternativas é obtida a partir de uma grandeza denominada fluxo líquido, que pode ser interpretada como uma pontuação atribuída à alternativa de acordo com o seu desempenho. 0 fluxo líquido é dado por (BRANS; VINCKE; MARESCHAL, 1986):

$$
Q(a)=Q^{+}(a)-Q^{-}(a)
$$

Uma alternativa a sobreclassifica uma alternativa $b$ se o fluxo líquido de $a$ for maior que o fluxo líquido de $b$, isto é, $Q(a)>Q(b)$, $a$ é indiferente a $b$ se seus fluxos líquidos forem iguais, isto é, $Q(a)=Q(b)$. A ordenação das alternativas é feita com base na ordem decrescente de seus respectivos fluxos líquidos.

A seção a seguir apresenta o contexto da decisão para fins de estruturar o problema que será apoiado pela MCDA.

\section{0 contexto da decisão}

O Senai, criado em 1942, por iniciativa do empresariado do setor industrial, é o maior complexo de educação profissional e tecnológica da América Latina. Ele é composto por um departamento nacional, que administra suas ações nacionais, e por 27 departamentos regionais, com unidades operacionais instaladas nos 26 estados e no Distrito 
Federal. Através das unidades operacionais, o Senai leva seus programas, projetos e atividades a todo o território nacional, oferecendo atendimento às diferentes necessidades locais e contribuindo para 0 fortalecimento da indústria e o desenvolvimento pleno e sustentável do país. Anualmente, mais de 2,5 milhões de trabalhadores brasileiros são qualificados através de 2.887 cursos, distribuídos em 7 modalidades: iniciação profissional; aprendizagem industrial; qualificação profissional; aperfeiçoamento profissional; técnico de nível médio; superiores de graduação e superiores de pós-graduação. Além dos cursos, o Senai também apoia empresas em 28 áreas industriais, por meio da qualificação de recursos humanos e da prestação de serviços técnicos e tecnológicos, como consultoria e assistência laboratorial, pesquisa aplicada e de informação tecnológica (BRASIL, 2012a).

0 Pronatec irá utilizar a infraestrutura do Senai, espalhada por todo o território nacional brasileiro, para expandir, interiorizar e democratizar a oferta de cursos de Educação Profissional e Tecnológica (EPT) no país. Com isso, o Senai estima que até 2014 o número de matrículas anuais aumente em $50 \%$, passando a 4 milhões de novas matrículas por ano. Só no estado da Paraíba, foram disponibilizadas, para o ano de 2012, 6.162 novas vagas para cursos profissionalizantes. Nas unidades do Senai de Campina Grande, isso será feito através de um conjunto de iniciativas, dentre elas a criação da bolsa-formação, que visa oferecer gratuitamente, por meio dos serviços nacionais de aprendizagem (Senai; Senac - Serviço Nacional de Aprendizagem Comercial; Senar -Serviço Nacional de Aprendizagem Rural; e Senat - Serviço Nacional de Aprendizagem do Transporte), cursos técnicos para estudantes matriculados no ensino médio e cursos de formação inicial e continuada ou qualificação profissional para grupos sociais de diferentes perfis. Dentro da iniciativa bolsa-formação serão oferecidas duas modalidades de curso: (i) cursos técnicos para quem está matriculado no ensino médio, com duração mínima de um ano; e (ii) cursos de formação inicial e continuada ou qualificação profissional, com duração mínima de dois meses. Além da bolsaformação, o Senai de Campina Grande também está apoiando o acordo de gratuidade com os serviços nacionais de aprendizagem, que tem por objetivo ampliar, progressivamente, a aplicação dos recursos dos serviços nacionais de aprendizagem, recebidos da contribuição compulsória, em cursos técnicos e de formação inicial e continuada ou de qualificação profissional, em vagas gratuitas destinadas a pessoas de baixa renda, com prioridade para estudantes e trabalhadores (BRASIL, 2012b; FEDERAÇÃO..., 2012).

Conforme a Lei n. 12.513/2011 (BRASIL, 2011), os critérios de seleção dos candidatos para essas iniciativas deverão priorizar as seguintes categorias: (i) estudantes do ensino médio da rede pública, inclusive da EJA; (ii) trabalhadores; (iii) beneficiários dos programas federais de transferência de renda; e (iv) estudante que tenha cursado o ensino médio completo em escola da rede pública ou em instituições privadas na condição de bolsista integral, nos termos do regulamento. A lei também estabelece a parceria entre União, estados, Distrito Federal e municípios para manutenção do programa, podendo cada região inserir mais critérios de seleção, de acordo com as modalidades de benefícios do programa e com as especificidades de cada região.

No que concerne à bolsa-formação para a primeira modalidade de curso, a Secretaria de Educação do Estado da Paraíba, em consonância com as diretrizes nacionais do Pronatec, determinou que os seguintes requisitos mínimos fossem atendidos pelos candidatos: os candidatos devem ter pelo menos 16 anos de idade; os candidatos devem estar matriculados nos $1^{\circ}, 2^{\circ} \mathrm{e}$ $3^{\circ}$ anos do ensino médio da rede pública de ensino ou da rede particular, desde que sejam beneficiários de bolsa de estudo integral, nos termos do regulamento; os candidatos devem possuir renda familiar baixa. Além disso, a secretaria determinou um conjunto de aspectos que devem ser priorizados durante a seleção dos candidatos: priorizar candidatos cuja idade distorça muito da idade média de alunos matriculados nessas séries; priorizar alunos com desempenho escolar bom; priorizar alunos que possuírem frequência regular às aulas; priorizar alunos que residam mais próximo à escola ofertante.

É com base nesses direcionamentos, que as unidades do Senai de Campina Grande-PB, atualmente selecionam os candidatos para a primeira modalidade de curso da bolsa-formação. No entanto, isso é feito de forma desestruturada, ou seja, não existe um procedimento que garanta a consideração de todos esses aspectos na avaliação de cada candidato. Além disso, a forma como se dá a avaliação de desempenho dos candidatos dentro de cada aspecto não é bem definida, o que pode fazer com que, para uma mesma lista de candidatos, o resultado da seleção seja diferente, de acordo com o profissional do Senai que participou do processo seletivo, revelando inclusive uma parcialidade na decisão.

Embora o Senai/Campina Grande esteja em consonância com as diretrizes nacionais do Pronatec, a forma como o processo seletivo é realizado não garante que as metas do programa sejam atendidas, no que concerne a abrangência que os benefícios terão na sociedade e à imparcialidade e transparência, fundamentais a esse tipo de iniciativa. Nesse sentido, a seção seguinte apresenta um procedimento para estruturar e apoiar a seleção de alunos do ensino 
médio para os cursos, com duração mínima de um ano, o qual é baseado em uma análise multicritério apoiada pelo método PROMETHEE 11.

\section{Procedimento proposto}

0 procedimento proposto visa apoiar as unidades do Senai/Campina Grande na seleção de candidatos à bolsa-formação do Pronatec, destinada a alunos do ensino médio para realizarem cursos no Senai, com duração mínima de um ano. Embora a proposta tenha sido elaborada para apoiar uma unidade específica do Senai, ela pode ser facilmente adaptada para apoiar outras unidades do Senai nos processos seletivos referentes à bolsa-formação do Pronatec, bem como a seleção referente a outras iniciativas do programa. Portanto, a descrição do procedimento é realizada de forma que o mesmo possa ser utilizado como modelo para apoiar outros processos seletivos decorrentes das iniciativas do Pronatec. 0 procedimento está dividido em cinco etapas (Figura 1).

A primeira etapa é destinada ao estabelecimento dos critérios, de acordo com as diretrizes do Pronatec e da Secretaria de Educação da Paraíba, bem como a definição dos pesos dos critérios de acordo com as preferências dos gestores do Senai/Campina Grande, responsáveis pelo processo seletivo. Durante a etapa de triagem, a documentação dos candidatos é verificada e é realizada uma pré-seleção com base nos critérios de seleção estabelecidos na primeira etapa. Na terceira etapa, os candidatos, que não foram eliminados na etapa de triagem, são avaliados segundo os critérios de avaliação estabelecidos. Então, o método multicritério PROMETHEE 11 é aplicado à matriz de avaliação de candidatos versus critérios, a fim de construir um ranking de candidatos com base em seus respectivos desempenhos globais. Finalmente, o resultado da seleção é divulgado na última etapa do procedimento.

\subsection{Estabelecimento dos critérios}

A realização dessa etapa requer a verificação das diretrizes que orientam a seleção de candidatos para as bolsas do Pronatec. Para as unidades do Senai/Campina Grande foram verificadas, além da legislação própria do programa, as recomendações da Secretaria de Educação do Estado referentes ao Pronatec. Espera-se que as recomendações no âmbito dos estados para o Pronatec, que no caso da Paraíba são feitas pela Secretaria de Educação do Estado, estejam em consonância com as demandas profissionais identificadas nas respectivas regiões, de modo a atribuir um caráter regional ao programa.

Os critérios estão divididos em duas categorias: (i) critérios de exclusão; e (ii) critérios de avaliação de desempenho. Os critérios de exclusão foram definidos de acordo com os requisitos mínimos estabelecidos, que devem ser atendidos pelos candidatos para concorrer ao benefício. Esses critérios são usados para determinar se um candidato pode ou não participar do processo de seleção. Para a primeira modalidade de curso da bolsa-formação, os critérios de exclusão são:

- Idade mínima permitida: só podem participar do processo seletivo candidatos com idade mínima igual a 16 anos; a verificação desse critério é feita a partir de documento de identidade entregue no ato da inscrição;

- Matrícula ativa no ensino médio da rede pública: só podem participar do processo seletivo candidatos que estejam devidamente matriculados nos $1^{\circ}, 2^{\circ}$ ou $3^{\circ}$ anos do ensino médio da rede pública de ensino ou da rede particular, desde que sejam beneficiários de bolsa de estudo integral; no caso dos candidatos da rede pública, a verificação desse critério é feita a partir de comprovante de matrícula regular que deve compor a lista de documentação exigida no ato da inscrição; já no caso dos candidatos da rede particular, além do comprovante de matrícula regular eles devem apresentar também declaração da escola que comprove o benefício de bolsa de estudo integral;

- Renda baixa: só podem participar do processo seletivo candidatos cuja renda familiar seja classificada como baixa, de acordo com os critérios adotados pelo Instituto Brasileiro de Geografia e Estatística (IBGE), que enquadra em baixa renda aquelas famílias cuja renda per capita é inferior a um salário mínimo; a verificação desse critério é feita a partir de comprovante de renda familiar.

Para a primeira modalidade de curso da bolsaformação foram estabelecidos cinco critérios de avaliação de desempenho, cujas descrições e escalas de avaliação estão apresentadas na Tabela 1.

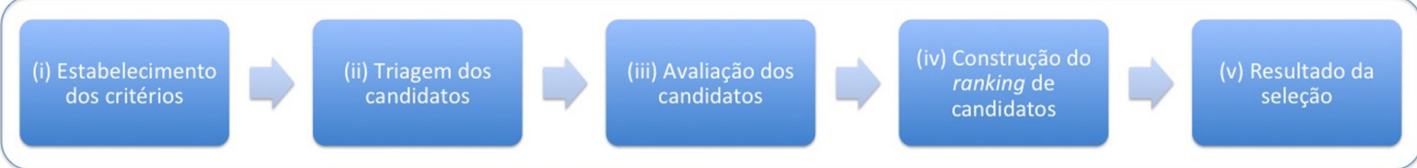

Figura 1. Fluxograma do modelo. 
Tabela 1. Critérios de avaliação de desempenho.

\begin{tabular}{|c|c|c|}
\hline ID & Nome do critério & Descrição \\
\hline $\mathrm{C} 1$ & Distorção idade-série & $\begin{array}{l}\text { Esse critério mede a distorção entre a idade dos candidatos e a série escolar, a qual o aluno está cursando. } \\
\text { A seleção irá priorizar os candidatos que apresentarem maior distorção idade-série. Assim, distorção idade- } \\
\text { série é critério de maximização, ou seja, quanto maior, melhor. Esse critério será avaliado através do padrão } \\
\text { de idade adotado para cada uma das séries da seguinte forma: para alunos cursando a } 1^{\text {a }} \text { série, subtrai da } \\
\text { idade do aluno } 16 \text { anos; para alunos cursando a } 2^{\text {a }} \text { série, subtrai da idade do aluno } 17 \text { anos; e para alunos } \\
\text { cursando a } 3^{\text {a }} \text { série, subtrai da idade do aluno } 18 \text { anos. }\end{array}$ \\
\hline $\mathrm{C} 2$ & Desempenho escolar & $\begin{array}{l}\text { Esse critério mede o desempenho escolar do aluno na série que ele está cursando. A seleção irá priorizar } \\
\text { candidatos com melhor desempenho escolar. Assim, desempenho escolar é critério de maximização, ou } \\
\text { seja, quanto maior, melhor. Esse critério será avaliado através do desempenho médio (média global) do } \\
\text { aluno em todas as disciplinas da série que ele está cursando. }\end{array}$ \\
\hline $\mathrm{C} 3$ & Frequência & $\begin{array}{l}\text { Esse critério mede a frequência do aluno na série que ele está cursando. A seleção irá priorizar candidatos } \\
\text { com melhor frequência escolar. Assim, frequência é critério de maximização, ou seja, quanto maior, melhor. } \\
\text { Esse critério será avaliado pela frequência do aluno na série escolar que ele está cursando e será dada em } \\
\text { percentual (\%). }\end{array}$ \\
\hline C4 & Proximidade à escola & $\begin{array}{l}\text { Esse critério mede a distância da residência dos candidatos à escola na qual o curso será ofertado. A } \\
\text { seleção irá priorizar candidatos que moram mais próximo à escola na qual o curso será ofertado. Assim, } \\
\text { proximidade à escola é critério de minimização, ou seja, quanto menor, melhor. A distância será verificada } \\
\text { pela ferramenta Google Maps e será dada em quilômetros }(\mathrm{km}) \text {. }\end{array}$ \\
\hline $\mathrm{C} 5$ & Renda & $\begin{array}{l}\text { Esse critério irá avaliar a renda familiar do candidato. A seleção irá priorizar candidatos com renda familiar } \\
\text { mais baixa. Assim, renda é critério de minimização, ou seja, quanto menor, melhor. Esse critério será } \\
\text { avaliado pela renda familiar per capita do candidato na unidade monetária real (R\$). }\end{array}$ \\
\hline
\end{tabular}

Após a definição do conjunto de critérios, o gestor da unidade na qual o curso será oferecido, ou responsável direto pelo processo de seleção, deve atribuir medidas de importâncias relativas aos critérios. Os pesos devem ser valores entre 0 e 10.0 peso de um critério irá corresponder à importância relativa desse critério dentro do processo seletivo, de modo que, quanto maior o peso, maior a importância atribuída pelo gestor ao critério. A atribuição de peso nulo para um dado critério não é permitida, pois implica na não consideração do respectivo critério dentro do processo decisório, o que não é permitido nesse caso, visto que a definição dos critérios é feita com base em diretrizes estabelecidas para o programa e que, portanto, devem ser obedecidas. Nesse sentido, também não são permitidos pesos com valores muito baixos que impliquem em uma contribuição irrelevante do critério na decisão final.

Em seguida, os pesos são normalizados dividindo cada um deles pela soma total dos pesos, de modo que a soma dos pesos normalizados seja igual a 1 .

Para a primeira modalidade de curso da bolsaformação, o Senai/Campina Grande estabeleceu o seguinte conjunto de pesos (Tabela 2).

Como a definição dos pesos dos critérios é feita com base nas preferências do gestor (ou responsável) direto das unidades, presumindo-se que essas preferências estejam em consonância com os interesses da organização, os valores podem ser alterados em diferentes seleções para o mesmo tipo de benefício, dentro da mesma unidade, desde que não sejam atribuídos pesos nulos ou valores muito baixos.

A flexibilidade na definição das medidas de importâncias relativas dos critérios é importante, pois
Tabela 2. Pesos dos critérios.

\begin{tabular}{lc}
\multicolumn{1}{c}{ Critério } & Peso \\
\hline Distorção idade/série & 0,2 \\
Desempenho escolar & 0,2 \\
Frequência regular & 0,2 \\
Proximidade à escola & 0,2 \\
Renda & 0,2 \\
\hline
\end{tabular}

permite fazer ajustes no processo seletivo conforme o desempenho do programa, sem comprometer o cumprimento de suas diretrizes básicas; por exemplo, caso seja identificado que o elevado número de evasão nas turmas seja devido à dificuldade dos alunos em frequentar as aulas, dada a distância de suas origens à unidade do Senai, onde esteja ocorrendo o curso, o critério "proximidade à escola" pode ter seu peso aumentado, de modo a priorizar a seleção de candidatos que residam mais próximo à unidade e, com isso, tentar resolver o problema da evasão escolar.

\subsection{Triagem dos candidatos}

Nessa etapa do procedimento, o setor responsável pelo processo de seleção irá avaliar a documentação entregue pelos candidatos a fim de verificar quais deles não atendem aos critérios de exclusão, seja pela ausência de propriedade referente aos requisitos mínimos ou pela ausência de documentação que os comprove. Os candidatos que não atenderem aos critérios de exclusão serão eliminados do processo de seleção. 0 nome desses candidatos, bem como o motivo da eliminação, serão divulgados ao final do processo juntamente com a lista dos candidatos selecionados. 


\subsection{Avaliação dos candidatos}

Nessa etapa, o setor responsável pelo processo de seleção irá avaliar a documentação entregue pelos candidatos a fim de preencher uma matriz de avaliação dos candidatos versus critérios de avaliação. Cada célula $p_{i j}$ irá conter o desempenho do candidato $i(i=1,2, \ldots, m)$ no critério $j(j=1,2, \ldots, n$, para essa aplicação $n=5$ ) (Figura 2). A avaliação deve ser realizada de acordo com as escalas de avaliação de cada critério, as quais foram definidas na primeira etapa do procedimento (Tabela 1).

0 candidato cuja documentação for insuficiente para realizar a avaliação em todos os critérios será eliminado do processo de seleção. 0 nome desse candidato assim como o motivo da eliminação serão divulgados ao final do processo juntamente com a lista dos candidatos selecionados.

\subsection{Construção do ranking de candidatos}

Nessa etapa, o método multicritério PROMETHEE 11 será usado para construir o ranking de candidatos de acordo com os desempenhos com relação aos critérios de avaliação considerados, os quais se encontram registrados na matriz de avaliação construída na etapa anterior.

Inicialmente, para cada critério $j$, é calculada a diferença de desempenho entre cada par de candidatos $(a, b)$, representada por $g_{j}(a)-g_{j}(b)$, que deve ser armazenada em uma matriz. Em seguida, para cada critério, deve ser atribuída uma das seis funções de preferências propostas por Brans, Vincke e Mareschal (1986) (Quadro 1).

A função de preferência de um critério irá mensurar a preferência por um candidato, com base na diferença de desempenho entre ele e os demais. Para os critérios $\mathrm{C} 1$, C2 e C3, ficou estabelecido que se o desempenho de um candidato a é ligeiramente maior que o desempenho de um candidato $b$, em um desses critérios, então o candidato $a$ é totalmente preferível ao candidato $b$. Matematicamente, tem-se: $P_{j}(a, b)=1$, se $g_{j}(a)-g_{j}(b)>0$ e $P_{j}(a, b)=0$, caso contrário. Portanto, para esses critérios, a função usual (tipo 1) é a mais apropriada.
Para o critério C4, que avalia o desempenho dos candidatos com base na proximidade com que eles residem da unidade onde o curso será realizado, ficou estabelecido que não há preferência de um candidato em relação a outro quando a diferença de desempenho nesse critério é de até $1 \mathrm{~km}$. Porém, quando essa diferença aumenta (acima de $1 \mathrm{~km}$ ), o candidato que mora mais próximo à unidade terá preferência sobre o que mora mais longe. Matematicamente, tem-se $P_{j}(a, b)=1$, se $g_{j}(a)-g_{j}(b)>1$ e $P_{j}(a, b)=0$, caso contrário. Portanto, para C4 a função mais apropriada é a função com formato U (tipo 11), com o limiar de indiferença $q$ igual a 1.

Para o critério $\mathrm{C} 5$, que avalia o desempenho dos candidatos com base em suas respectivas rendas familiares, ficou estabelecido que não há preferência de um candidato em relação a outro quando a diferença de desempenho nesse critério é de até $R$ \$ 50. Porém, quando essa diferença é maior que $R \$ 50$, o candidato com renda familiar menor terá preferência sobre o outro. Matematicamente, tem-se $P_{j}(a, b)=1$, se $g_{i}(a)-g_{i}(b)>50$ e $P_{i}(a, b)=0$, caso contrário. Portanto, a função com formato U (tipo II) também é a mais apropriada para $\mathrm{C} 5$, sendo que, nesse caso, o limiar de indiferença $q$ deve ser igual a 50 .

0 próximo passo é calcular o índice de preferência para cada par de candidatos, $P(a, b)$, usando a equação 1 , que agrega as intensidades de preferência de um candidato sobre outro considerando todos os critérios. Para essa aplicação o índice de preferência é dado por:

$$
\begin{aligned}
& P(a, b)=p_{1} P_{1}(a, b)+p_{2} P_{2}(a, b)+p_{3} P_{3}(a, b) \\
& +p_{4} P_{4}(a, b)+p_{5} P_{5}(a, b)
\end{aligned}
$$

onde $p_{1}, p_{2}, p_{3}$, $p_{4}$ e $p_{5}$ são os pesos, já normalizados, dos critérios $\mathrm{C} 1, \mathrm{C} 2, \mathrm{C} 3$, C4 e $\mathrm{C}$, respectivamente.

Os valores devem ser armazenados em uma matriz de candidatos versus candidatos. Finalmente, os fluxos positivos, negativos e líquidos são calculados, conforme Equações 3, 4 e 5, respectivamente. 0 fluxo positivo pode ser interpretado como a soma de todas as vantagens de um candidato sobre os demais. Por outro lado, o fluxo negativo seria a soma de suas desvantagens. Portanto, os fluxos líquidos representam medidas de desempenho global

\begin{tabular}{l|c|c|c|c|c}
\hline Candidatos & $\begin{array}{c}\text { Distorção } \\
\text { idade/série }\end{array}$ & $\begin{array}{c}\text { Desempenho } \\
\text { escolar }\end{array}$ & $\begin{array}{c}\text { Frequência } \\
\text { regular }\end{array}$ & $\begin{array}{c}\text { Proximidade } \\
\text { à Escola }\end{array}$ & Renda \\
\hline & & & & & \\
\hline & & & & & \\
\hline & & & & & \\
\hline & & & & & \\
\hline
\end{tabular}

Figura 2. Matriz candidatos versus critérios de avaliação. 
do candidato e serão usados para ordená-los. Um candidato $a$ terá prioridade sobre um candidato $b$ se $Q(a)>Q(b)$. Caso dois candidatos tenham o mesmo fluxo líquido $Q(a)=Q(b)$ fica caracterizado o empate entre eles. Os casos de empate devem ser resolvidos de acordo com critérios de desempate estabelecidos para o processo decisório. Para essa aplicação, será priorizado o candidato mais velho; se o empate persistir, será priorizado o candidato com renda familiar mais baixa.

\subsection{Resultado da seleção}

No resultado final do processo seletivo, três situações possíveis são atribuídas a cada um dos candidatos inscritos: (i) classificado e aprovado; (ii) classificado; ou (iii) desclassificado. A primeira situação é atribuída aos candidatos da $1^{\text {a }}$ até a $k$-ésima posição do ranking obtido com o método PROMETHEE, onde $k$ é o número total de bolsas ofertadas pela instituição. A segunda situação é atribuída aos candidatos das posições $k+1$ até $2 k$ do ranking, indicando que 0 processo classifica um número de candidatos que é igual ao dobro da quantidade de bolsas ofertadas. Isso significa que os candidatos dessas posições podem ser contemplados com a bolsa quando houver disponibilidade, decorrente da desistência de algum candidato aprovado, obedecendo sempre a ordem de prioridade indicada pelo ranking. A situação desclassificado é atribuída aos demais candidatos do ranking e aos candidatos eliminados do processo nas etapas de pré-seleção e avaliação dos candidatos.

0 resultado final do processo seletivo deve ser divulgado na página oficial da instituição e das demais organizações envolvidas no processo, assim como nos principais jornais impressos de circulação no município no qual a seleção foi realizada. A documentação referente ao processo seletivo deve ser arquivada na instituição e também enviada para as demais organizações envolvidas.

A sessão seguinte apresenta uma aplicação do procedimento e uma análise comparativa entre o resultado de uma seleção obtido sem o uso do procedimento e o resultado da seleção obtido com o procedimento. A aplicação foi realizada sobre os dados dos candidatos que se submeteram à seleção realizada pelo Senai em 2011 para o curso cortador de calçados.

\section{Aplicação}

A seleção para o curso cortador de calçados, realizada por uma das unidades do Senai no município de Campina Grande-PB, no ano de 2011, resultou em uma lista de 30 candidatos, onde os 15 primeiros foram contemplados com bolsa-formação do Pronatec, destinada a alunos do ensino médio. Embora a seleção tenha sido realizada em consonância com os princípios do programa, não há garantia de que todos os critérios estabelecidos pelo Pronatec em suas diretrizes, bem como aqueles decorrentes das recomendações da Secretaria de Educação da Paraíba, tenham sido efetivamente atendidos, visto que não havia uma estruturação para o problema de seleção, nem tampouco um método adequado para apoiar o processo.

0 procedimento proposto, que contempla a estruturação do problema e um método multicritério para apoiar a seleção propriamente dita, foi aplicado ao mesmo conjunto de dados, isto é, aos dados referentes aos 31 candidatos que se submeteram à seleção para o curso cortador de calçados do Senai/ Campina Grande em 2011. Com relação às importâncias atribuídas aos critérios, foi considerado o conjunto de pesos da Tabela 2. A Tabela 3 apresenta a lista dos 15 primeiros candidatos obtida com o processo de seleção convencional e com o processo de seleção utilizando o procedimento proposto.

Os resultados mostram diferenças bastante significativas entre as duas formas de realizar a seleção para o conjunto de dados utilizados. Com o procedimento, $45 \%$ das vagas teriam sido ocupadas por outros candidatos. Outra diferença considerável diz respeito ao primeiro colocado na seleção convencional, o candidato A16, que não teria ficado dentro das 15 primeiras vagas caso tivesse sido usado o procedimento. Da mesma forma os primeiros colocados da seleção com o procedimento proposto ( $\mathrm{A} 12$ e $\mathrm{A} 1)$ não foram selecionados dentro do número de vagas no processo convencional. Apenas o último colocado (A30) coincidiu nos dois casos.

Tabela 3. Candidatos selecionados.

\begin{tabular}{ccc} 
& \multicolumn{2}{c}{ Candidatos } \\
\cline { 2 - 3 } Posição & $\begin{array}{c}\text { Seleção } \\
\text { convencional }\end{array}$ & $\begin{array}{c}\text { Seleção com o } \\
\text { procedimento }\end{array}$ \\
\hline $1^{\text {a }}$ & A16 & A12 \\
$2^{\text {a }}$ & A17 & A1 \\
$3^{\text {a }}$ & A18 & A22 \\
$4^{\text {a }}$ & A19 & A11 \\
$5^{\text {a }}$ & A20 & A25 \\
$6^{\text {a }}$ & A21 & A10 \\
$7^{\text {a }}$ & A22 & A18 \\
$8^{\text {a }}$ & A23 & A14 \\
$9^{\text {a }}$ & A24 & A17 \\
$10^{\text {a }}$ & A25 & A20 \\
$11^{\text {a }}$ & A26 & A5 \\
$12^{\text {a }}$ & A27 & A3 \\
$13^{\text {a }}$ & A28 & A29 \\
$14^{\text {a }}$ & A29 & A19 \\
$15^{\text {a }}$ & A30 & A30 \\
\hline
\end{tabular}


Avaliando os desempenhos dos candidatos A16, A12 e A1 em cada critério individualmente, é possivel inferir sobre a efetividade do procedimento: no critério distorção idade/série, que é de maximização, o desempenho do candidato A12 foi 13, bastante superior ao desempenho de $A 1$ e $A 16$, que ficaram com 1 e 0 , respectivamente; no critério desempenho escolar (critério de maximização), o desempenho de A12 foi levemente inferior ao de A1 (A12 ficou com $7,23$ e $A 1$ com 7,3$)$, enquanto o desempenho de A16 foi significativamente inferior $(5,87)$; no critério proximidade à escola (critério de minimização), o desempenho de $\mathrm{A} 16$ foi 44 , drasticamente inferior aos desempenhos de $\mathrm{A} 12$ e $\mathrm{A} 1$, que ficaram com 3,4 e 1,8, respectivamente; nos demais critérios, os desempenhos dos três candidatos foram bastante similares.

A análise justifica o mau desempenho global do candidato A16 no ranking obtido com o procedimento proposto e, consequentemente, o bom desempenho dos candidatos $\mathrm{A} 12$ e A1, que ocuparam, respectivamente, a primeira e a segunda posições nesse ranking. Com relação aos candidatos $\mathrm{A} 12$ e A1, especificamente, o primeiro teve um desempenho muito melhor sobre o segundo no critério distorção idade/série; já nos demais critérios avaliados, o desempenho de A12 foi inferior ao de $A 1$, porém com diferenças pouco significativas, o que levou $\mathrm{A} 12$ a ocupar a primeira posição do ranking. Essa análise é um indicativo da qualidade do procedimento proposto no que concerne ao atendimento às diretrizes do Pronatec no âmbito do Estado da Paraíba.

0 procedimento mostrou-se robusto à mudança nas importâncias relativas dos critérios. Foi feita uma outra simulação do procedimento, atribuindo-se um peso maior para o critério renda $\left(p_{5}=0,4\right)$ e atribuindo-se pesos iguais $\left(p_{1}=p_{2}=p_{3}=p_{4}=0,15\right)$ para os demais critérios. 0 resultado se manteve, com exceção dos candidatos A13 e A6, que tiveram suas posições invertidas.

Também foi feita uma simulação, atribuindo-se uma importância relativa baixa ao critério $\mathrm{C} 3$, que mede a frequência escolar dos candidatos, visto que as informações fornecidas pelos candidatos referentes a esse critério podem não ser muito confiáveis. A nova configuração dos pesos ficou: $p_{1}=p_{2}=p_{4}=0,2$; $p_{3}=0,1$; e $p_{5}=0,3$. 0 resultado se manteve igual ao da simulação anterior.

Como o procedimento prevê alterações nos parâmetros pesos em diferentes seleções para o mesmo tipo de benefício, dentro da mesma unidade, essa análise é importante para evidenciar a flexibilidade que o gestor da unidade tem em interferir no processo de seleção, mesmo com o uso do procedimento, que é inflexível quanto aos aspectos que devem ser levados em consideração na seleção.

\section{Conclusão}

Este estudo teve como objetivo a proposição de um procedimento para apoiar as unidades do Senai em Campina Grande nos processos de seleção de alunos do ensino médio para os cursos de educação profissional e tecnológica disponibilizados pelo governo federal através do Pronatec.

Para avaliar e priorizar os candidatos, o procedimento utilizou uma abordagem multicritério, onde a definição dos critérios foi feita com base nas diretrizes do Pronatec e nas recomendações para o programa estabelecidas no âmbito do estado da Paraíba, visando atribuir um caráter regional ao programa. Nesse sentido, é importante que essas recomendações estejam em consonância com demandas profissionais identificadas para cada região. Consequentemente, o procedimento melhora a qualidade do processo de seleção, pois garante que as metas do Pronatec sejam efetivamente atendidas e que os candidatos selecionados atendam também ao perfil demandado por esse tipo de formação e pelo setor industrial local.

A estruturação do procedimento permitiu também apoiar todas as etapas do processo de seleção dos candidatos, incluindo a atividade de divulgação do resultado final, o que é de fundamental importância para garantir a imparcialidade e transparência do processo seletivo, que são essenciais em decisões cujas consequências têm impacto direto sobre a sociedade. Uma possível consequência disso é a redução de conflitos decorrentes da insatisfação de candidatos não aprovados na seleção.

Outra importante contribuição deste trabalho é permitir que o procedimento proposto seja utilizado como modelo para apoiar unidades do Senai de outros estados da federação. Essa utilização pode ocorrer tanto para apoiar na seleção de estudantes para a bolsa-formação como também para apoiar outras iniciativas do Pronatec, tal como a bolsa-formação do trabalhador.

\section{Referências}

AlMEIDA, A. T. Processo de Decisão nas Organizações: Construindo Modelos de Decisão Multicritério. São Paulo: Editora Atlas, 2013.

ALMEIDA, A. T.; COSTA, A. P. C. S. Modelo de decisão multicritério para a priorização de sistemas de informação com base no método PROMETHEE. Gestão e Produção, v. 9, p. 201-214, 2002. http://dx.doi.org/10.1590/ S0104-530X2002000200007

ALMEIDA, A. T.; VETSCHERA, R. A note on scale transformations in the PROMETHEE V method. European Journal of Operational Research, v. 219, n. 1, p. 198200, 2012. http://dx.doi.org/10.1016/j.ejor.2011.12.034 
BANA E COSTA, C. A.; CORTE, J. M.; VANSNICK, J. C. On the mathematical foundations of MACBETH. In: FIGUEIRA, J.; GRECO, S.; EHRGOTT, M. (Ed.). Multiple criteria decision analysis: state of the art surveys. New York: Springer, 2005. p. 409-442.

BEHZADIAN, M. et al. PROMETHEE: A comprehensive literature review on methodologies and applications. European Journal of Operational Research, v. 200, n. 1, p. 198-215, 2010. http://dx.doi.org/10.1016/j. ejor.2009.01.021

BELTON, V.; STEWART, T. J. Multiple Criteria Decision Analysis. Kluwer Academic Publishers, 2002. http:// dx.doi.org/10.1007/978-1-4615-1495-4

BRANS, J. P.; VINCKE, P. A preference ranking organization method (The PROMETHEE method for multiple criteria decision-making). Management Science, v. 31, p. 647656, 1985. http://dx.doi.org/10.1287/mnsc.31.6.647

BRANS, J. P.; VINCKE, P.; MARESCHAL, B. How to select and how to rank projects: The PROMETHEE method. European Journal of Operational Research, v. 24, p. 228-238, 1986. http://dx.doi.org/10.1016/03772217(86)90044-5

BRASIL. Lei Ordinária $n^{\circ}$ 12.513, de 26 de outubro de 2011. Institui o Programa Nacional de Acesso ao Ensino Técnico e Emprego (Pronatec). Diário Oficial da República Federativa do Brasil, Brasília, DF, 27 out. 2011. Disponível em: <https://www.planalto.gov.br/ccivil_03/_ato20112014/2011/lei/112513.htm>. Acesso em: 05 mar. 2012.

BRASIL. Serviço Nacional de Aprendizagem Industrial. $O$ que é o Senai? Brasília, 2012a. Disponível em: <http://www. portaldaindustria.com.br>. Acesso em: 8 mar. 2012.

BRASIL. Ministério da Educação. Qualificação técnica e profissional para trabalhadores e alunos do ensino médio. Brasília: Ministério da Educação, 2012b. Disponível em: <http://pronatecportal.mec.gov.br/bolsa.html> Acesso em: 8 mar. 2012.

EDWARD, W.; BARRON, F. H. SMARTS and SMARTER: Improved simple methods for multiattribute utility measurement. Organizational Behaciour and Human Decision Processes, v. 60, p. 306-325, 1994. http:// dx.doi.org/10.1006/obhd.1994.1087

FEDERAÇÃO DAS INDÚSTRIAS DO ESTADO DA PARAÍBA - FlEPB. Notícias. Campina Grande, 2012. Disponível em <http://www.fiepb.com.br>. Acesso em: 05 mar. 2012.
KEENEY, R. L.; RAIFFA, H. Decision with Multiple Objectives: Preference and Value Trade-offs. New York: Wiley, 1976.

LEYVA-LOPEZ, J. C. Multicriteria Decision Aid Application to a Student Selection Problem. Pesquisa Operacional, v. 25, n. 1, p. 45-68, 2005. http://dx.doi.org/10.1590/ S0101-74382005000100004

MUNDA, G. Social multi-criteria evaluation for a sustainability economy. Berlin: Springer-Verlag, 2008. http://dx.doi. org/10.1007/978-3-540-73703-2

RANGEL, L. A. D.; GOMES, L. F. A. M. 0 Apoio Multicritério à Decisão na avaliação de candidatos. Produção, v. 20, n. 1, p. 92-101, 2010. http://dx.doi.org/10.1590/S010365132010005000016

ROY, B. Methodologie Multicrière d'aide à la Décision. Paris: Editora Econômica, 1985.

SAATY, T. L. The Analytic Hierarchy Process. New York: McGraw-Hill, 1980.

SCHRAMM, F.; MORAIS, D. C. Decision support model for selecting and evaluating suppliers in the construction industry. Pesquisa Operacional, v. 32, n. 3, p. 643-662, 2012. http://dx.doi.org/10.1590/S010174382012005000020

SILVA, V. B. S.; MORAIS, D. C.; ALMEIDA, A. T. A Multicriteria Group Decision Model to Support Watershed Committees in Brazil. Water Resources Management, v. 24, n. 14, p. 4075-4091, 2010. http://dx.doi.org/10.1007/s11269010-9648-2

VETSCHERA, R.; ALMEIDA, A. T. A PROMETHEE-based approach to portfolio selection problems. Computers \& Operations Research, v. 39, n. 5, p. 1010-1020, 2012. http://dx.doi.org/10.1016/j.cor.2011.06.019

VIANA, J. C.; ALENCAR, L. H. Metodologias para seleção de fornecedores: uma revisão da literatura. Produção, v. 22 , n. 4, p. 625-636, 2012. http://dx.doi.org/10.1590/ S0103-65132012005000067

VINCKE, P. Multicriteria decision aid. Bruxelles: Jonh Wiley \& Sons, 1992.

\section{Agradecimentos}

Este trabalho é resultado do Curso de Especialização em Gestão da Organização Pública financiado pela Universidade Aberta do Brasil (UAB)/Coordenação de Aperfeiçoamento de Pessoal de Nível Superior (CAPES).

\title{
The use of the PROMETHÉE method for the selection of candidates to Pronatec's scholarship
}

\begin{abstract}
The objective of this study is the construction of a procedure to aid the SENAl in Campina Grande, Paraiba, in the selection of high school students for enrollment in professional and technology courses provided by the federal government through the PRONATEC program. The procedure includes the establishment of exclusion and selection criteria according to the PRONATEC guidelines and some rules established by the Secretary of Paraíba for Education; a structured evaluation of candidates, supported by the multicriteria method PROMETHÉE 11; and the structuring of the selection process disclosure. The procedure improves the quality of the selection process, ensuring that the program's goals are met.
\end{abstract}

\section{Keywords}

Multicriteria analysis. PROMETHEE. Candidate selection. PRONATEC. 